\title{
Relato de experiência: terapia nutricional enteral domiciliar - promoção do direito humano à alimentação adequada para portadores de necessidades alimentares especiais
}

\section{Experience report: home enteral nutrition therapy - promoting the human right to adequate food for people with special dietary needs}

Ann Kristine Jansen'

Kimielle Cristina Silva²

Gilberto Simeone Henriques'

Janete dos Reis Coimbra ${ }^{3}$

Maria Tereza Gouveia Rodrigues ${ }^{4}$

Ana Maria dos Santos Rodrigues ${ }^{5}$

Suellen Fabiane Campos ${ }^{4}$

Simone de Vasconcelos Generoso

1 Departamento de Nutrição, Escola de Enfermagem. Universidade Federal de Minas Gerais. Belo Horizonte, MG, Brasil.

${ }^{2}$ Coordenação Geral de Alimentação e Nutrição, Departamento de Atenção Básica, Secretaria de Atenção à Saúde, Ministério da Saúde. Belo Horizonte, MG, Brasil.

${ }^{3}$ Coordenação dos Núcleos de Apoio a Saúde da Família, Secretaria Municipal de Saúde de Belo Horizonte, Minas Gerais. Belo Horizonte, MG, Brasil.

${ }^{4}$ Secretaria Municipal de Saúde de Belo Horizonte, Minas Gerais. Belo Horizonte, MG, Brasil.

${ }^{5}$ Programa de Pós-graduação em Enfermagem, Escola de Enfermagem. Universidade Federal de Minas Gerais. Belo Horizonte, MG, Brasil.

Apoio financeiro: Fundação de Amparo à Pesquisa de Minas Gerais (FAPEMIG) Edital 07/2012, Processo №. CDS-APQ-02570-12 Ministério da Saúde/Organização Pan-Americana da Saúde, Carta Acordo BR/LOA/1300093

Correspondência / Correspondence Ann Kristine Jansen

Av Professor Alfredo Balena, 190, Santa Efigênia. Escola de Enfermagem, Sala 314 30130-100, Belo Horizonte, MG, Brasil E-mail: aki@enf.ufmg.br

\section{Resumo}

Trata-se de relato da experiência vivenciada pelo projeto desenvolvido pelo Grupo de Trabalho em Terapia Nutricional Enteral Domiciliar de Belo Horizonte, Minas Gerais. Este grupo tem parceria com dez hospitais públicos do município e com a Secretaria Municipal de Saúde de Belo Horizonte. O objetivo do projeto é qualificar a atenção nutricional ofertada pelo Sistema Único de Saúde (SUS). Os resultados já alcançados foram: um fluxo de alta hospitalar dos indivíduos em nutrição enteral; a atualização em terapia enteral domiciliar para os nutricionistas do Núcleo de Apoio à Saúde da Família; uma cartilha para cuidador do paciente em terapia enteral domiciliar; e a análise química de macronutrientes e minerais das dietas enterais artesanais prescritas na alta hospitalar. Estão em andamento a análise química de vitaminas da dieta; a elaboração de um manual técnico em terapia nutricional enteral domiciliar para os profissionais da Atenção Básica e a criação de material educativo digital para educação à distância. O projeto já conquistou um encaminhamento assistido dos pacientes de alta hospitalar para Atenção Básica; equipes mais bem treinadas e capacitadas no manejo e atenção da terapia nutricional enteral domiciliar, e disponibilizará fórmulas enterais artesanais de baixo custo e fácil preparo, de composição química conhecida, beneficiando milhares de indivíduos e promovendo o direito humano à alimentação adequada para portadores de necessidades alimentares especiais.

Palavras-chave: Segurança Alimentar e Nutricional. Terapia Nutricional. Nutrição Enteral. Assistência Domiciliar. Sistema Único de Saúde. Adultos. 


\section{Abstract}

This is an experience report of a project developed by the Working Group on Home Care Enteral Nutrition Therapy of Belo Horizonte, Minas Gerais state. The partners of this project are ten public hospitals and the Municipal Health Department of Belo Horizonte. The aim was to qualify the nutritional home care offered by the Health Unified System (SUS). To date, the results were a routine of hospital discharge of individuals under enteral nutrition, refresher courses on home care enteral therapy to nutritionists from the Family Health Support Center, a handbook for caregivers of patients under home enteral therapy and chemical analysis of macronutrients and minerals from homemade enteral diets prescribed after hospital discharge. The next steps will be the chemical analysis of vitamins of the homemade diet, the design of a technical handbook on home enteral nutrition therapy for primary care professionals and the creation of digital learning material for distance education. Outcomes of the project include an assisted referral of hospital discharge patients to primary care and better trained and qualified staff in the management and care of home enteral nutrition therapy. Soon, the project will provide low cost and easyto-prepare homemade enteral formulas with known chemical composition, benefiting thousands of individuals and promoting the human right to adequate food for people with special dietary needs.

Key words: Food Security and Nutrition. Nutritional Therapy. Enteral Nutrition. Home Care. Health System. Adults.

\section{Contextualização da experiência}

O alargamento do topo da pirâmide etária, com o aumento da participação relativa da população com 65 anos ou mais, de 5,9\% em 2000 para 7,4\% em 2010, associado a maior expectativa de vida, ${ }^{1}$ impacta nos processos de saúde, com elevação da prevalência de doenças associadas à idade, como as doenças crônicas não transmissíveis (DCNT) e as neurodegenerativas. Este fenômeno tem levado a uma reorganização do sistema de saúde no Brasil. ${ }^{2}$

O Plano de Ações para Enfrentamento das DCNTs do Ministério da Saúde ${ }^{2}$ destaca, entre outras metas, o cuidado integral e a promoção da saúde, cujas principais ações incluem a capacitação das equipes de Atenção Básica, com investimentos na telemedicina e ampliação da atenção no domicílio a pessoas que precisam de cuidados regulares ou intensivos, mas não de hospitalização, como idosos debilitados com estabilidade clínica e indivíduos acamados. 
Nesse contexto, os cuidados em terapia nutricional enteral no domicílio são cada vez mais comuns, principalmente em portadores de agravos que resultam na falência oral, como o câncer de cabeça, pescoço ou esôfago e as doenças e desordens neurológicas. ${ }^{3,4,5}$ Frequentemente, nestas situações clínicas, a terapia nutricional enteral (TNE) e uso de fórmulas nutricionais se fazem necessários por períodos prolongados, como forma de preservar ou recuperar o estado nutricional. ${ }^{3}$

A TNE no domicílio é uma alternativa viável e vantajosa, pois promove o convívio dos indivíduos com familiares, melhorando o conforto e a qualidade de vida. ${ }^{5,6}$ Além disso, contribui para a garantia do direito à alimentação adequada e saudável aos portadores de necessidades alimentares especiais, seguindo as diretrizes e objetivos da Política Nacional de Alimentação e Nutrição (PNAN) e do Sistema Nacional de Segurança Alimentar e Nutricional (SISAN). ${ }^{8}$

A PNAN define como prioritárias as ações preventivas e de tratamento da obesidade, da desnutrição, das carências nutricionais específicas e de doenças crônicas não transmissíveis relacionadas à alimentação e nutrição. Mas também aponta como demanda para a atenção nutricional no Sistema Único de Saúde (SUS), o cuidado aos indivíduos que apresentam necessidades alimentares especiais. ${ }^{7}$

Sabe-se que existem inúmeras doenças e agravos à saúde que, assim como as próprias fases do curso da vida, podem causar mudanças nas necessidades alimentares de cada indivíduo. As necessidades alimentares especiais são definidas na PNAN como:

As necessidades alimentares, sejam restritivas ou suplementares, de individuos portadores de alteração metabólica ou fisiológica que cause mudanças, temporárias ou permanentes, relacionadas à utilização biológica de nutrientes ou a via de consumo alimentar (enteral ou parenteral). ${ }^{7}$

Neste sentido, portadores de necessidades alimentares especiais são considerados indivíduos vulneráveis à insegurança alimentar e nutricional, que necessitam de políticas de promoção do Direito Humano à Alimentação Adequada. ${ }^{8}$

A TNE no domicílio é uma terapêutica conhecida, presente nos Estados Unidos há mais de 25 anos. No Brasil, a partir de estudos do Grupo de Apoio Nutricional Enteral e Parenteral no período de 1990-1999, observou-se aumento de 64\% da indicação de TNE. ${ }^{9}$ Dados da Sociedade Britânica de Nutrição Parenteral e Enteral mostram que, ao final de 2007, havia 21.858 pessoas em TN domiciliar, representando aumento de $11,6 \%$ em um ano. ${ }^{10}$

A transferência do paciente do hospital para o domicílio requer uma estrutura familiar adequada, treinamento e assistência do cuidador, pois este deverá assumir a responsabilidade sobre todas as tarefas relacionadas ao preparo e administração da dieta e cuidados com o 
paciente e a sonda. ${ }^{11-13}$ Muitos familiares de indivíduos em TNE relatam a transferência para o domicílio como positiva, por ser uma oportunidade de proximidade do familiar. ${ }^{5}$ No entanto, demonstram vulnerabilidade, medo do futuro, ansiedade, insegurança, isolamento na nova função e impossibilidade de lazer e felicidade junto ao paciente como sentimentos ambivalentes. ${ }^{12-14}$ Assim, esta família necessita da assistência de equipes treinadas e capacitadas no manejo desse tipo de atenção domiciliar, considerando a alta complexidade do cuidado destes indivíduos. ${ }^{5,6,11-13}$

No centro da discussão do tema do cuidado ofertado a indivíduos em TNE no domicílio está a dieta. Segundo a Agência Nacional de Vigilância Sanitária (ANVISA) e de acordo com a Resolução RDC n ${ }^{\circ} 63$, de 6 de julho de 2000, a dieta na nutrição enteral é todo alimento, para fins especiais, formulada e elaborada para uso em cateteres ou via oral, podendo ser industrializada ou não, sendo utilizada em regime hospitalar, ambulatorial ou domiciliar. ${ }^{15}$

Há uma diversidade de formulações industrializadas no mercado e a vantagem da maioria destas são a composição nutricional, a osmolalidade e a estabilidade adequadas, além da segurança microbiológica. ${ }^{16}$ No âmbito hospitalar, são utilizadas e custeadas pelo SUS apenas em hospitais habilitados de acordo com a Portaria SAS/MS no 120, de 14 de abril de 2009..$^{17}$ Assim, quando há indicação de TNE no domicílio por longo período, há orientação do uso de fórmulas artesanais, ou semiartesanais, que nem sempre são nutricionalmente adequadas. ${ }^{16,18}$ Muitas destas fórmulas são compostas por alimentos in natura disponíveis no domicílio, diluídos, liquidificados e peneirados, sem padronização das medidas caseiras, dos procedimentos de preparo e do tempo de cocção, comprometendo a composição nutricional..$^{19} \mathrm{~A}$ falta de padronização pode aumentar o risco de desnutrição e comorbidades, como pneumonias, anemia, infecção do trato respiratório e urinário, ${ }^{20,21}$ além de contribuir para a reinternação hospitalar. ${ }^{9}$

Na literatura são escassos os trabalhos que propõem dietas enterais artesanais completas nutricionalmente, de baixo custo, com estabilidade e osmolalidade apropriadas. Há algumas propostas adequadas em macronutrientes, mas ainda com raros estudos quanto aos micronutrientes. ${ }^{22,23}$ A maioria dos estudos de composição química das dietas enterais artesanais utiliza tabelas de composição química, sem levar em conta as perdas que ocorrem devido ao processamento dos alimentos. ${ }^{24}$ Há escassez de estudos bromatológicos e experimentais que quantifiquem precisamente os macro e micronutrientes, garantindo aporte nutricional adequado ao longo do tratamento, evitando o surgimento de deficiências nutricionais.

Outro problema das dietas enterais artesanais domiciliares refere-se a sua segurança microbiológica, pela necessidade de controle das condições higiênicas de preparo, acondicionamento e administração. ${ }^{15}$ Estudo recente encontrou $24 \%$ das amostras de dietas enterais artesanais contaminadas com bactérias mesófilas e $36 \%$ com coliformes fecais, ${ }^{22}$ alertando os nutricionistas das equipes de saúde para a necessidade de corrigirem falhas na manipulação por parte do cuidador, orientando e acompanhando o preparo das dietas nos domicílios. 
Os vários fatores relacionados à dieta, desde sua prescrição até o preparo no domicílio, apontam para a necessidade de introdução e qualificação dessa temática nos cursos de graduação em Nutrição, assim como o investimento nos processos de educação permanente para os nutricionistas que atuam nos serviços de saúde.

O custo, argumento frequentemente utilizado na defesa de dietas artesanais, nem sempre justifica a indicação, pois algumas formulações artesanais disponíveis na literatura apresentam valor similar ao de dietas industrializadas. ${ }^{16}$

Entende-se, pois, que se faz necessário o exercício da técnica dietética aplicada e do estudo experimental da matriz alimentar para que se determine com grau analítico a composição de dietas artesanais e se ratifique suas aplicações na TNE no domicílio, associada a uma proposta de baixo custo e de fácil preparo para reduzir os erros provenientes de medidas caseiras e o risco de contaminação no processo de preparo, armazenamento e administração da dieta.

Desta forma, com o objetivo de qualificar a atenção nutricional ofertada no âmbito da atenção domiciliar pelo SUS no Município de Belo Horizonte-MG, foi desenvolvido o projeto "Terapia Nutricional Enteral Domiciliar - promoção do direito humano à alimentação adequada para portadores de necessidades alimentares especiais", na perspectiva de contribuir para a garantia do direito humano à alimentação adequada de grupos portadores de necessidades alimentares especiais.

\section{Método}

Este artigo consiste em um relato da experiência vivenciada pelo Grupo de Trabalho em Terapia Nutricional Domiciliar de Belo Horizonte, Minas Gerais.

Nos hospitais públicos deste município, a alta de indivíduos com alimentação por via alternativa sempre foi uma problemática para as equipes de suporte nutricional hospitalar e, em particular, para os nutricionistas. Os principais pontos de conflito eram o elevado custo da dieta enteral industrializada, a reduzida qualidade de muitas dietas artesanais, o baixo poder aquisitivo da maioria dos indivíduos atendidos pelo SUS e o pouco tempo disponível na alta para ensinar a família do paciente no preparo e administração da dieta no domicílio. De forma adicional, havia a dificuldade das Equipes de Saúde da Família (ESF), dos Núcleos de Apoio à Saúde da Família (NASF) e do Programa de Atenção Domiciliar (PAD) no cuidado a estes indivíduos, e a ausência de fluxos de referência e contrarreferência entre os níveis de atenção à saúde. Esses fatores dificultavam a resolução de problemas decorrentes desta via de alimentação, resultando na debilitação e desnutrição do paciente, piora da qualidade de vida e frequente reinternação hospitalar. 
Assim, em meados de 2009, criou-se o Grupo de Trabalho em Terapia Nutricional Domiciliar, com a participação de professores do Curso de Nutrição da Universidade Federal de Minas Gerais (UFMG), de nutricionistas do NASF e de nutricionistas de três hospitais públicos de Belo Horizonte. As primeiras atividades do grupo foram o desenvolvimento de um fluxo de alta hospitalar dos indivíduos em nutrição enteral, a promoção de um curso de atualização em TNE no domicílio para os nutricionistas do NASF e a elaboração de uma cartilha para o cuidador do paciente. No entanto, estas atividades ainda não atingiam a maior parte dos indivíduos e cuidadores, pois a adesão dos hospitais públicos do município era baixa, o que prejudicava os objetivos do grupo.

Neste sentido, perante a problemática exposta, apresentou-se à Fundação de Amparo à Pesquisa de Minas Gerais (FAPEMIG) proposta de ampliação do projeto, segundo Edital 07/2012, e realizouse um trabalho de sensibilização junto aos demais hospitais públicos de Belo Horizonte, que se tornaram parceiros do projeto. A estratégia utilizada nessa sensibilização foi a possibilidade da análise química das dietas enterais artesanais prescritas na alta e a implementação do fluxo de alta, com referência para os nutricionistas do NASF, garantindo a atenção nutricional domiciliar e reduzindo o risco de reinternações. Esse trabalho possibilitou a adesão de $90 \%(n=10)$ dos hospitais públicos $(n=11)$ de atendimento a indivíduos adultos existentes na cidade. Excluíram-se unidades hospitalares públicas de especialidades, como ortopedia, oftalmologia, infectologia, e as maternidades, onde a alta com via de alimentação enteral é muito incomum.

Ainda ao final de 2011, com a entrada de novos nutricionistas nas equipes do NASF da Secretaria Municipal de Saúde de Belo Horizonte, a coordenação da Área de Nutrição da SMS trouxe a demanda de realizar um novo projeto de atualização dos nutricionistas em cuidados em TNE.

O projeto apresentado à FAPEMIG foi aprovado, sendo seu financiamento liberado no início de 2013, e contemplou as seguintes atividades: atualização dos nutricionistas recém-contratados no NASF; elaboração de um manual em Terapia Nutricional Domiciliar para Equipes de Saúde da Família; elaboração de aula de educação à distância (EAD), a ser veiculada no Programa Telessaúde/UFMG para os nutricionistas dos NASF dos demais municípios mineiros; e análise de macronutrientes e minerais das dietas enterais artesanais de uso domiciliar prescritas pela Atenção Básica do município e pelos hospitais parceiros do projeto.

Em meados de 2013, a partir da divulgação dos resultados do projeto em seminários regionais e encontros nacionais, a Coordenação Geral de Alimentação e Nutrição (CGAN) do Ministério da Saúde (MS) propôs a ampliação do projeto, com análise de vitaminas das dietas e expansão do material educativo para educação à distância, por acreditar que a problemática da terapia nutricional enteral domiciliar vivenciada pelos nutricionistas dos NASFs de Belo Horizonte é realidade no restante do Brasil, e também pela inexistência desse tipo de conteúdo 
dirigido aos nutricionistas da Atenção Básica e Atenção Domiciliar. Assim, elaborou-se a FASE II do projeto, que foi financiada pelo MS por meio de Carta-Acordo com a Organização Panamericana da Saúde (OPAS).

O projeto tem previsão de término em fevereiro de 2015; portanto, no presente artigo, apresentaremos os resultados obtidos até o momento, na tentativa de contribuir com outras experiências em andamento.

Sem dúvida, os fatores que potencializaram o desenvolvimento deste projeto foram o tema em questão, a quantidade de dúvidas e incertezas quanto a dietas artesanais prescritas nas altas hospitalares, os custos envolvidos na prescrição inadequada e falência na recuperação ou estabilização dos indivíduos em TNE. Por outro lado, a proposta do estudo das dietas artesanais hospitalares impossibilitou a adesão de $100 \%$ dos hospitais, com receio de perda de autonomia ou imposição de uma ou outra dieta na prescrição de alta. Ao longo de toda sensibilização, sempre houve a transparência de que o grupo não pretende impor dietas de alta hospitalar, mas, a partir do conhecimento da composição química das dietas atualmente prescritas, será possível propor fórmulas artesanais de melhor composição e conhecer os nutrientes que eventualmente será necessário suplementar.

\section{Resultados}

\section{Fluxograma de encaminhamento de indivíduos em uso de TNE}

A primeira atividade do grupo de trabalho foi o desenvolvimento e instituição de um fluxo de referência (figura 1), entre os hospitais envolvidos no projeto e o NASF. O fluxo estabeleceu que todo indivíduo morador de Belo Horizonte, em alta hospitalar (dos hospitais participantes do grupo) com via alternativa de alimentação e dieta enteral, deve ser encaminhado à Unidade Básica de Saúde (UBS) de referência com relatório de alta contendo o histórico nutricional. O Serviço Social do hospital ou o próprio nutricionista responsável pela orientação da dieta de alta entrará em contato com o Serviço Social da UBS ou do NASF de referência, avisando da necessidade de visita domiciliar para acompanhamento clínico e nutricional. Foi estabelecido que a visita domiciliar inicial será realizada pela ESF, que solicitará o acompanhamento do nutricionista do NASF de referência, estabelecendo-se um acompanhamento multidisciplinar, se necessário.

Esse fluxo proporcionou o estabelecimento de uma rede de atenção, ainda incipiente, em que pedidos e compromissos estão sendo pactuados. Segundo Mendes, ${ }^{25}$ este pacto ocorre em função de seu "comum" investindo na produção de saúde, produção de autonomia e valorização dos agentes participantes do processo e a sustentabilidade do processo. 


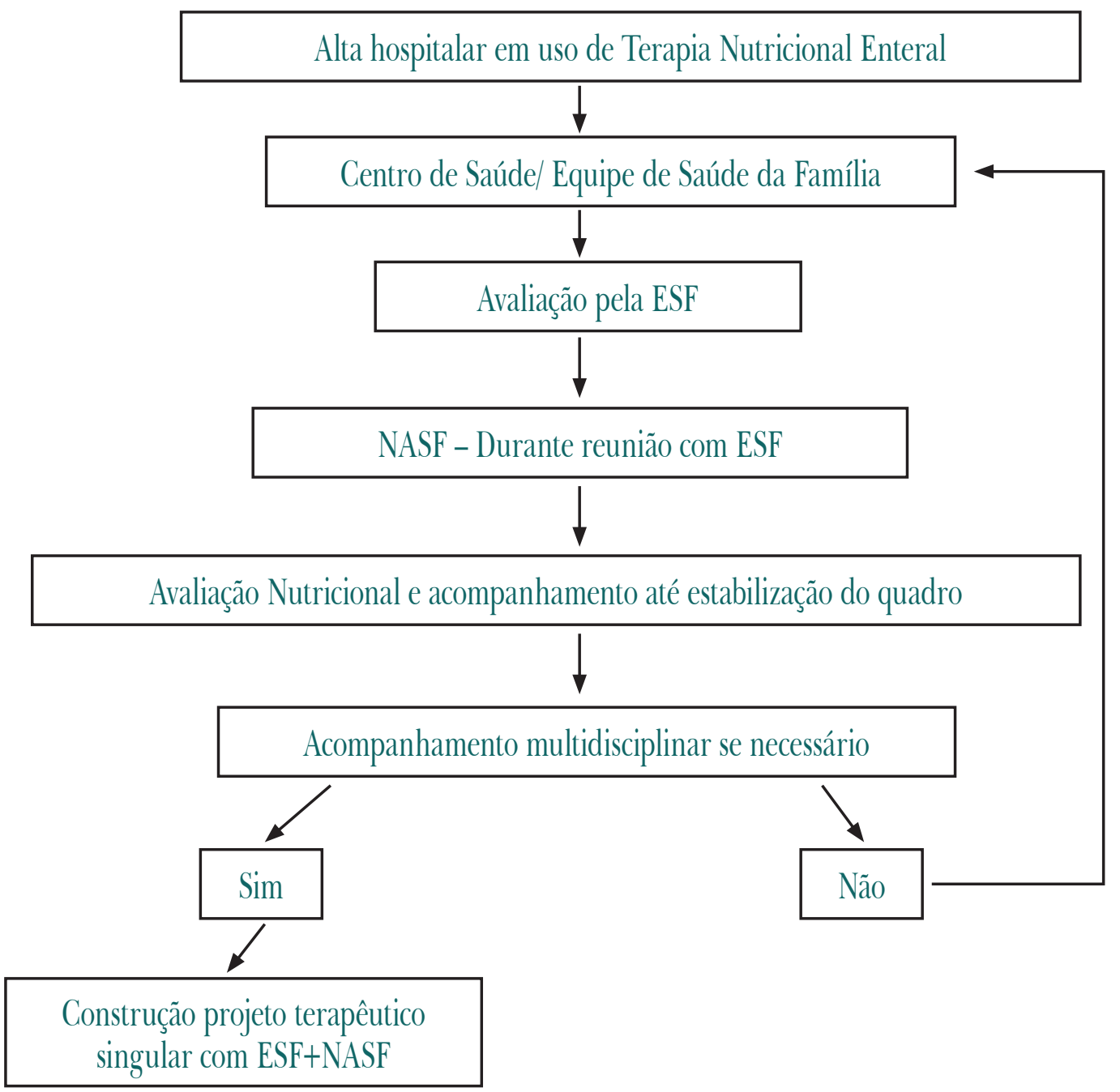

Figura 1. Fluxograma de encaminhamento de indivíduos em uso de TNE. 


\section{Atualização dos nutricionistas do NASF}

O estabelecimento do fluxo de encaminhamento dos indivíduos em TNE exigiu atualização profissional dos nutricionistas do NASF da SMS, pois estes apresentavam habilidades e competências adquiridas ao longo de sua graduação ou em atuação profissional em nutrição clínica hospitalar, e não na vivência desta situação na Atenção Básica e domiciliar. Assim, realizaram-se duas capacitações, ambas com 30 horas de duração. A primeira, em 2010, contou com a participação de 41 nutricionistas, e a segunda, ainda em andamento e com previsão para término em outubro de 2014, com mais 27 nutricionistas do NASF sendo capacitados.

Devido à mudança no quadro de profissionais em 2012/2013, com a criação de novas vagas e a substituição de contratos temporários por efetivos, apenas $15,1 \%(n=8)$ dos nutricionistas capacitados em 2010 permaneceram na rede. Então, 100\% (universo total=41) dos nutricionistas do NASF foram capacitados na primeira etapa e 50,9\% (universo total =53) na segunda. Assim, ao final da segunda atividade de atualização, 66,0\% (n=35) dos nutricionistas do NASF-BH estarão capacitados no manejo do paciente em nutrição enteral domiciliar.

A abordagem desta atualização envolve encontros presenciais realizados na Escola de Enfermagem da UFMG, com abordagem multiprofissional. Os temas propostos são ministrados por profissionais médicos, enfermeiros, farmacêuticos, psicólogos e nutricionistas dos hospitais parceiros e do NASF. Os principais assuntos discutidos foram relativos ao cuidado do indivíduo, preparo e manipulação da dieta, cuidados com a sonda e identificação e controle de complicações. Além das discussões teóricas, a metodologia de ensino inclui estudos de caso e simulações em laboratórios de práticas de enfermagem e de técnica dietética.

As dietas artesanais da rotina de orientação de alta dos hospitais públicos envolvidos no projeto foram preparadas pelos nutricionistas do NASF, com apoio dos autores e alunos bolsistas do projeto, no laboratório de dietética. Em seguida, foram realizados testes de fluidez e viscosidade, administrando-se as dietas pelo método gravitacional, com auxílio de equipo e em bolus, por meio de seringas de $20 \mathrm{ml}$, por sondas nasogástricas e nasoentéricas para indivíduos adultos, calibre 12. A estabilidade das dietas também foi testada por inspeção visual do processo de separação de fases em período de 12 horas de armazenamento. 
Após a primeira capacitação em 2010, houve um momento de alinhamento de técnicas de avaliação nutricional e outros temas referentes a atividades do nutricionista na atenção básica, no qual foram discutidas as dúvidas e dificuldades da prescrição da dieta artesanal. Além disso, o acompanhamento desses profissionais ocorreu por meio de discussão contínua de casos com a referência técnica de nutrição da Secretaria de Saúde. A avaliação do impacto da atividade de atualização em andamento no momento será realizada no seminário final, que ocorrerá em outubro de 2014, e na prática diária, em conjunto com a referência técnica de nutrição da Secretaria de Saúde.

\section{Cartilha de orientações em terapia nutricional enteral domiciliar para o cuidador}

Como produto da primeira atualização dos nutricionistas, elaborou-se, no âmbito da atividade de capacitação, com a participação de todos os nutricionistas parceiros do projeto, uma cartilha de orientações em TNE no domicílio. O material foi impresso pela Secretaria de Saúde do município, sendo entregue pelos nutricionistas do NASF ao cuidador na primeira visita domiciliar ao paciente em alimentação enteral. Contém informações sobre tipos de dieta, cuidados no preparo da dieta enteral, conservação da dieta, modos de administração da dieta, limpeza da sonda, hidratação do paciente, procedimentos em situações de entupimento da sonda, diarreia, constipação intestinal, náuseas e vômitos, estase gástrica e saída da sonda. Ainda informa ao cuidador sobre administração de medicamentos e outros cuidados com o paciente.

Ao final da cartilha, é apresentada uma proposta de dieta artesanal com formulações de $1.500,1.800$ e 2.100 quilocalorias e opção padrão ou para hiperglicemia, onde o nutricionista deve assinalar qual formulação a ser seguida. A dieta proposta foi desenvolvida por Espósito e colaboradores, ${ }^{26}$ e sua composição química, analisada por meio de tabelas, atende a muitas das recomendações nutricionais de adultos homens de 50-71 anos. ${ }^{27}$ Além disso, é de fácil preparo e baixo custo (custo diário de $\mathrm{R} \$ 5,57$ para a dieta $1.800 \mathrm{Kcal}$ padrão e de $\mathrm{R} \$ 5,95$ para a dieta $1.800 \mathrm{Kcal}$ para hiperglicemia). Esta dieta tem sido prescrita por dois dos hospitais parceiros do projeto e pelo NASF de Belo Horizonte.

Apesar de a cartilha não ter sido testada antes de sua implementação na rede, o fato de a maioria dos nutricionistas responsáveis pela entrega do material no domicílio ter participado ou estar participando da capacitação em terapia nutricional enteral domiciliar permite, em caso de dúvidas do cuidador, o esclarecimento e a adequada orientação. Assim, a cartilha é um material de apoio ao cuidador, e não o único informativo sobre a dieta e sua administração. 


\section{Análise das dietas}

A análise bromatológica sempre foi um grande impeditivo à segurança da administração de dietas enterais artesanais, tornando sua composição precisa motivo de grandes dúvidas e discussão, fazendo com que esse fosse um argumento largamente utilizado por fabricantes de dietas enterais industrializadas para seu desuso ou descontinuidade, em detrimento de fórmulas nas quais indubitavelmente a rotulagem e seus aspectos legais garantem o conteúdo de nutrientes e supostamente a harmonia da combinação desses componentes. Prementemente sobre a dieta artesanal, por sua preparação caseira, tem-se um menor controle da qualidade nutricional e microbiológica.

A análise química das dietas pretende assegurar aos profissionais e usuários da terapia nutricional enteral domiciliar o uso de dietas altamente especializadas, com composição definida. Além disso, a elaboração de fichas técnicas de preparo de fácil entendimento auxilia no uso eficiente e seguro na rotina da TNE no domicílio.

Assim, 11 dietas artesanais de alta hospitalar prescrita por oito hospitais públicos, além da prescrita pelo NASF, foram estudadas quanto a viscosidade, estabilidade, custo e técnica de preparo. Destas, foram selecionadas cinco para análise, pois atendiam à proposta de uma dieta artesanal de baixo custo, fácil preparo, sem necessidade de serem peneiradas para evitar a retenção de boa parte dos nutrientes, e com viscosidade e estabilidade adequadas, capazes de serem administradas tanto em bolus (com auxílio de seringa de $20 \mathrm{ml}$ ) quanto pelo método gravitacional (com auxílio de equipo), por sonda nasogástrica ou nasoentérica calibre 12.

A análise da composição centesimal de macronutrientes foi realizada pelos métodos tradicionais de análise preconizados pela Association of Analytical Chemists, e de minerais, pela técnica de espectrometria de emissão atômica. De forma global, encontraram-se boa adequação de macronutrientes e baixa quantidade de fibras, cobre, magnésio e manganês. Três dietas apresentaram quantidade elevada de sódio. Os demais minerais estavam com quantidades adequadas em comparação com as recomendações para homens de 50 a 71 anos. ${ }^{27}$ Os resultados detalhados dessas análises estão sendo publicados em outro artigo deste número temático. A adequação de sódio já foi realizada e a dos demais minerais e da fibra está sendo estudada.

Ainda serão analisadas as vitaminas e a osmolalidade. Após a finalização e avaliação dos resultados obtidos por meio das análises, novas dietas serão propostas e divulgadas por meio de aula de educação à distância e publicações.

As próximas duas atividades ainda estão em desenvolvimento, por isso serão brevemente descritas para que o leitor possa conhecer a totalidade do projeto. 


\section{Elaboração do Manual Técnico em Terapia Nutricional Enteral Domiciliar}

A elaboração do Manual Técnico em Terapia Nutricional Enteral Domiciliar se faz importante para o estabelecimento de protocolos baseados na literatura e em conhecimentos técnico-científicos, que padronizem as etapas do atendimento nutricional ao paciente em uso de TN no domicílio. Desta forma, pretende-se criar uma ferramenta de apoio para que os nutricionistas vinculados ao NASF e os profissionais das Equipes de Saúde da Família tenham autonomia no desenvolvimento dos processos de assistência ao paciente em TNE, de forma a garantir a continuidade e a integralidade da atenção a esses indivíduos.

Assim, o objetivo do manual é constituir uma referência técnico-científica de consulta e esclarecimento aos profissionais da Atenção Básica, além da padronização do atendimento nutricional ao paciente em uso de TNE no domicílio. Ele está sendo elaborado com a participação dos profissionais que ministraram as aulas na capacitação aos nutricionistas sob supervisão dos pesquisadores.

Serão impressos exemplares para os nutricionistas do NASF, as Equipes de Saúde da Família, considerando um exemplar para cada equipe das Unidades Básicas de Saúde (UBS) e para cada equipe do Programa de Atenção Domiciliar (PAD) de Belo Horizonte.

\section{Elaboração de material educativo digital para Educação à Distância (EAD)}

A proposta de elaborar material educativo para educação à distância ocorreu por se acreditar que a problemática da TNE no domicílio vivenciada pelos nutricionistas do NASF de Belo Horizonte deve ser mais intensa em municípios de pequeno e médio porte de Minas Gerais e do Brasil e, também, pela inexistência desse tipo de conteúdo dirigido aos nutricionistas de NASF, nos Programas de Telessaúde da UFMG. Assim, propõe-se criar material educativo digital dispondo de processos que extrapolem a mera superação da distância física, criando um conjunto de mídias nas quais os conteúdos se apresentem de forma dialógica e contextualizada, favorecendo uma aprendizagem significativa.

O material será uma mídia eletrônica, acompanhada de vídeo com demonstração da manipulação e preparo da dieta enteral artesanal, incluindo receitas, fichas de preparação e valor nutricional. Também incluirá orientações que devem ser repassadas ao cuidador, como: administração da dieta, cuidados com a sonda, higienização, conservação da dieta no domicílio, 
hidratação, situações problema (diarreia, vômito, estase, náusea, saída da sonda etc.), além de situações práticas da TNE, vivenciadas por nutricionistas do NASF de Belo Horizonte, como forma de contextualização e discussão de problemas enfrentados no domicílio.

O material será disponibilizado à CGAN/MS para ser veiculado na RedeNutri, aprimorando a qualificação dos nutricionistas dos NASF do Brasil nessa temática.

\section{Perspectivas}

O projeto fortalece a relação da universidade com os gestores e os profissionais do SUS em prol da melhoria da atenção nutricional na Rede de Atenção à Saúde do município, de forma a garantir o Direito Humano à Alimentação Adequada e Saudável aos indivíduos em terapia nutricional enteral no domicilio.

Por meio desse trabalho, conquistou-se um fluxo de encaminhamento assistido dos pacientes de alta hospitalar para a Atenção Básica; equipes mais bem treinadas e capacitadas no manejo e atenção da terapia nutricional enteral domiciliar. O projeto contribuirá para a produção e a difusão de conhecimento científico sobre o tema "dietas enterais artesanais", ainda incipiente na literatura, o que dará subsídios para as práticas de formação de profissionais, tanto na graduação quanto na educação permanente em serviços de saúde.

A experiência desenvolvida em Belo Horizonte apresenta metodologia aplicável a outros municípios brasileiros, e o apoio do Ministério da Saúde contribuirá para este compartilhamento, induzindo o aprimoramento do cuidado aos indivíduos em terapia nutricional enteral no domicílio ofertado pelo SUS e fortalecendo a implementação da PNAN no Brasil.

\section{Agradecimentos}

Agradecemos à Fundação de Amparo à Pesquisa de Minas Gerais (FAPEMIG) e ao Ministério da Saúde, pelo apoio financeiro para realização deste estudo. Agradecemos também a todos os parceiros não autores ou financiadores deste projeto - Serviço de Nutrição e Dietética do Hospital das Clínicas da Universidade Federal de Minas Gerais; Serviço de Nutrição e Dietética do Hospital Risoleta Tolentino Neves; Fundação Hospitalar de Minas Gerais (FHEMIG); Hospital Municipal Odilon Behrens, Instituto de Previdência e Saúde do Estado de Minas Gerais (IPSEMG); Secretaria Municipal de Saúde de Belo Horizonte e Núcleo de Apoio à Saúde da Família -, pela parceria, e pela formatação e impressão das cartilhas. 


\section{Referências}

1. Instituto Brasileiro de Geografia e Estatística. Estatísticas demográficas do Censo 1991, 2000 e 2010. Disponível em URL:http://www.ibge.gov.br/home/download/estatistica.shtm

2. Brasil. Ministério da Saúde. Plano de ações estratégicas para enfrentamento das DCNT no Brasil 2011-2022. Brasília: Ministério da Saúde; 2011. Disponível em: URL:http://portal.saude.gov.br/ portal/saude/profissional/area.cfm?id_area $=1818$

3. Fogg L. Home enteral feeding part1: an overview. British Journal of Community Nursing 2006; 12(6): 246-52.

4. De Luis DA, et al. Experience over 12 years with home enteral nutrition in a healthcare area of Spain. Journal of Human Nutrition and Dietetics 2013; 26(1):39-44.

5. Cabrit R, Lambert T, Simard M, Cavayé M, Creste M, Dumery S, et al. Nutrition entérale à domicile: 3 millions de journées d'expérience. Nutrition Clinique et Métabolisme 2013; 27(4):178-184.

6. Planas M, Pérez-Portabella C, Rodriguez T, Puiggrós C, Elvira D, Dalmau E. Evaluación del grado de satisfacción de un programa de nutrición enteral domiciliaria patient satisfaction in a home enteral nutrition program. Nutrición Hospitalaria 2007; 22(5):612-15.

7. Brasil. Ministério da Saúde. Política Nacional de Alimentação e Nutrição. Brasília: Ministério da Saúde; 2012.

8. Câmara Interministerial de Segurança Alimentar e Nutricional. Plano Nacional de Segurança Alimentar e Nutricional, 2012/2015. Brasília: Ministério do Desenvolvimento Social e Combate à Fome, CAISAN; 2011.

9. Borges VC, et al. Nutrição domiciliar: uma experiência no Brasil. In: Waitzberg DL. Nutrição oral, enteral e parenteral na prática clínica. 3. ed. Rio de Janeiro: Atheneu; 2002. p. 977-81.

10. Jones B. Artificial nutrition support in the UK 2000-2006: a report by the British Artificial Nutrition Survey (BANS), a committee of BAPEN (The British Association of Parenteral and Enteral Nutrition). BAPEN; 2008. Disponível em: URL:http://www.bapen.org.uk/pdfs/bans_reports/bans_report07.pdf.

11. Madigan SM, Fleming P, McCann S, Wright ME, MacAuley D. General practitioners involvement in enteral tube feeding at home: a qualitative study. BMC Family Practice 2007; 8(29):1-7.

12. Best C, Hitchings H. Enteral tube feeding: from hospital to home. British Journal of Nursing 2010; 19(3):174-9.

13. Bjuresäter K, Larsson M, Athlin E. Struggling in an inescapable life situation: being a close relative of a person dependent on home enteral tube feeding. J. Clin. Nursing 2012; 21(7-8):1051-1059.

14. Liley AJ, Manthorpe J. The impact of home enteral tube feeding in everyday life: a qualitative study. Health and Social Care in the Community 2003; 11(5):415-22.

15. Brasil. Resolução RDC no 63 de 6 de julho de 2000. Aprova regulamento técnico para fixar os requerimentos mínimos exigidos para a terapia nutricional enteral. Diário Oficial da União, 07 jul. 2000. 
16. Borghi R, Dutra Araujo T, Airoldi Vieira RI, Theodoro de Sousa T, Waitzberg DL. ILSI Task Force on enteral nutrition; estimated composition and costs of blenderized diets. Nutrición Hospitalaria 2013; 28(6):2033-2038.

17. Brasil, Ministério da Saúde. Secretaria de Atenção à Saúde. Portaria no 120 de 14 de abril de 2009. Diário Oficial da União, 20 abr. 2009; seção 1(74):72.

18. Sullivan MM, Sorreda-Esguerra P, Platon MB, Castro CG, Chou NR, Shott S, et al. Nutritional analysis of blenderized enteral diets in the Philippines. Asia Pacific Journal of Clinical Nutrition 2004; 13(4):385-91.

19. Menegassi B, Santana LS, Coelho JC, Martins OA, Pinto JPAN, Costa TMB, et al. Características físico-químicas e qualidade nutricional de dietas enterais não-industrializadas. Alimentos e Nutrição 2007; 18(2):127-32.

20. Santos VFNS, Morais TB. Nutritional quality and osmolality of homemade enteral diets, and followup of growth of severely disabled children receiving home enteral nutrition therapy. J. Trop. Pediatr 2009; 56(2):127-128.

21. Klek S, Szybinski P, Sierzega M, Szczepanek K, Sumlet M, Kupiec M, et al. Commercial enteral formulas and nutrition support teams improve the outcome of home enteral tube feeding. J. Parenter. Enteral. Nutr 2011; 35(3):380-385.

22. Santos VFN, Bottoni A, Morais TB. Qualidade nutricional e microbiológica de dietas enterais artesanais padronizadas preparadas nas residências de pacientes em terapia nutricional domiciliar. Revista de Nutrição 2013; 26(2):205-14.

23. Sousa LRM, Ferreira SMR, Schieferdecker MEM. Physicochemical and nutritional characteristics of handmade enteral diets. Nutrición Hospitalaria 2014; 29:568-574.

24. Henriques GS, Rosado GP. Formulação de dietas enterais artesanais e determinação da osmolalidade pelo método crioscópico. Revista de Nutrição 1999; 12(3):225-32.

25. Mendes EV. As redes de atenção à saúde. Revista Medica de Minas Gerais. 2008; 18(4S4):S3-11.

26. Espósito JS; Soares ADN, Jansen AK. Desenvolvimento de uma dieta enteral artesanal para uso domiciliar. Anais Congresso Brasileiro de Nutrição Enteral e Parenteral; 2009; Natal. Natal: SBNPE; 2009. p. 66.

27. Institute of Medicine. Dietary Reference Intakes. The essential guide to nutrient requirements. Washington DC: The National Academies Press; 2006. 543 p. 
